\title{
Análisis Morfométrico del Axis para la Fijación Atlantoaxial
}

\author{
Morphometric Analysis of the Axis for Atlantoaxial Fixation
}

\author{
Rodolfo Morales-Avalos ${ }^{1}$; Francisco Ismael Villarreal-Garcia ${ }^{1}$; Priscila Madelein Requena-Araujo ${ }^{1}$; \\ Félix Vílchez-Cavazos ${ }^{3}$; Pedro Martin Reyes-Fernándezi1; Oscar Armando Martínez-Gutierrez; \\ Victor Manuel Peña-Martínez ${ }^{1}$; Santos Guzmán-López ${ }^{1}$ \& Rodrigo E. Elizondo-Omaña ${ }^{1}$
}

MORALES-AVALOS, R.; VILLARREAL-GARCIA, F. I.; REQUENA-ARAUJO, P. M.; VÍLCHEZ-CAVAZOS, F.; REYESFERNÁNDEZ, P. M.; MARTÍNEZ-GUTIERREZ, O. A.; PEÑA-MARTÍNEZ, V. M.; GUZMÁN-LÓPEZ, S. \& ELIZONDOOMAÑN, R. E. Análisis morfométrico del axis para la fijación atlantoaxial. Int. J. Morphol., 36(4):1331-1336, 2018.

RESUMEN: El objetivo de este estudio fue evaluar de manera integral los parámetros morfométricos de la vértebra axis (C2) en la población mexicana involucrados en la realización de procedimientos quirúrgicos con el fin de proveer datos cuantitativos indispensables en su abordaje quirúrgico. Para este estudio se utilizaron un total de 576 vértebras axis $(\mathrm{C} 2)$ de población mexicana contemporánea. Las mediciones de las vértebras se efectuaron bilateralmente utilizando un vernier digital milimétrico con una precisión de $0.01 \mathrm{~mm}$ (Mitutoyo Digimatic w/Absolute Encoders- Series 500). Un total de 576 vértebras axis (C2), fueron medidas de manera bilateral, todas nuestras mediciones se reportaron en $\mathrm{mm}$. El promedio del ancho del pedículo fue de 8,96 mm con una DE $\pm 2,11 \mathrm{~mm}$. El promedio de la altura del pedículo fue de 10,82 $\mathrm{mm}$ con una DE de $\pm 1,89 \mathrm{~mm}$. El promedio de la altura del proceso odontoideo fue de $16,90 \mathrm{~mm}$ con una DE de $\pm 2,99 \mathrm{~mm}$. El promedio del ancho del proceso odontoideo fue de 9,99 $\mathrm{mm}$ con una de DE de $\pm 0,80 \mathrm{~mm}$. El promedio del diámetro de la faceta articular fue de $8,44 \mathrm{~mm}$ con una DE de $\pm 1,04 \mathrm{~mm}$. El promedio del diámetro AP del cuerpo vertebral fue de 15,11 $\mathrm{mm}$ con una DE de $\pm 1,88 \mathrm{~mm}$. El promedio del diámetro trasverso del cuerpo vertebral fue de 17,93 $\mathrm{mm}$ con una DE de $\pm 2,22 \mathrm{~mm}$. El promedio de la altura del cuerpo vertebral fue de 18,54 $\mathrm{mm}$ con una DE de 2,38 $\mathrm{mm}$. El promedio de la altura de las láminas fue de 11,53 $\mathrm{mm}$ con una DE de $\pm 1,39 \mathrm{~mm}$. El promedio del ancho de las láminas fue de $6,10 \mathrm{~mm}$ con una DE de $\pm 1,44 \mathrm{~mm}$. Los resultados obtenidos en nuestras mediciones demuestran una variación con los resultados de otros autores en diferentes estudios de piezas osteológicas y de estudios de imagen del axis (C2), lo que sugiere, con el fin de reducir los riesgo de daño a estructuras neurovasculares, utilizar técnicas y medidas especiales para la estabilización atlantoaxial de la población mexicana.

PALABRAS CLAVE: Anatomía; Morfometria; Axis, C2; Unión atlantoaxial; México.

\section{INTRODUCCIÓN}

El complejo atlantoaxial (C1-C2), presenta estructura compleja, se compone de las 2 vértebras superiores de la columna cervical, sus superficies articulares y sus estructuras ligamentarias. La estabilidad de este segmento es proporcionada principalmente por ligamentos debido a la falta de discos intervertebrales (Fried et al., 1973).

Condiciones cervicales degenerativas, cirugía cervical previa, anomalías congénitas de columna cervical y traumatismos pueden llegar a ocasionar inestabilidad atlantoaxial (Finn et al., 2008).

El complejo atlantoaxial presenta más de la mitad del movimiento rotatorio del craneo con respecto a la columna torácica (Penning \& Wilmink, 1987). Debido a esto, existe una gran falla para la inmovilización en esta región, en com- paración con el resto de la columna cervical (Fried et al.). Rupturas del los ligamentos: transverso (Heller et al., 1993), alar (Panjabi et al., 1991) y apical, fracturas del proceso odontoideo y odontoidectomías (Dickman et al., 1995) son las 3 causas comunes de desestabilización atlantoaxial que se han estudiado biomecánicamente en la actualidad.

En los últimos años se ha registrado un aumento en la incidencia de fracturas del proceso odontoideo, las cuales actualmente representan un $15 \%$ de todas las fracturas de la columna cervical.

Factores como la enfermedad de base y los efectos adversos del tratamiento empleado para la misma, pueden llegar a complicar el manejo de pacientes con inestabilidad de la union atlantoaxial.

\footnotetext{
${ }^{1}$ Módulo de Columna, Servicio de Ortopedia y Traumatología, Hospital Universitario “Dr. José Eleuterio González”, Universidad Autónoma de Nuevo León (U.A.N.L.), Monterrey, Nuevo León, México.

${ }^{2}$ Departamento de Anatomía Humana, Facultad de Medicina, Universidad Autónoma de Nuevo León (U.A.N.L.), Monterrey, Nuevo León, México.
} 
Hasta las últimas décadas, el tratamiento para la inestabilidad atlantoaxial era la inmovilización externa. La tasa de no unión y la morbilidad asociada con esta técnica han limitado su uso.

El alambrado posterior y las técnicas de injerto, como las técnicas de Gallie, Brooks-Jenkins y Sonntag, han sido utilizadas para lograr artrodesis del segmento en el manejo de la inestabilidad atlantoaxial (Dickman et al., 1991).

La fijación transarticular es la técnica de elección para los procedimientos de inestabilidad de la columna cervical secundarios a inestabilidad atlantoaxial. Esta técnica es útil para lograr estabilidad biomecánica con un bajo porcentaje de complicaciones (Jeanneret \& Magerl, 1992). Dentro de las complicaciones, se pueden presentar lesiones de la arteria vertebral y fallas en el material de osteosintesis como migración de material o ruptuta del mismo (Hanson et al., 1991).

Las características morfométricas de las vértebras determinan el tamaño de los tornillos a utilizar tanto en diámetro como en longitud, así como la forma, dirección y angulación ideal del tornillo al momento de su colocación (Morales-Avalos et al., 2012).

Es importante para el cirujano, el conocimiento de las características morfométricas para evitar lesiones de la corteza pedicular, meninges, raíces nerviosas, nervios craneales, facetas articulares, medula espinal o la arteria vertebral debido a la colocación u orientación incorrecta de los tornillos (Morales-Avalos et al.).

El objetivo del presente estudio fue determinar las características morfométricas de relevancia quirúrgica de la vértebra axis (C2) en las técnicas más utilizadas en la actualidad para la estabilización quirúrgica del complejo atlantoaxial.

\section{MATERIAL Y MÉTODO}

Estudio anatómico, observacional, transversal y descriptivo. La muestra consistió en un total de 576 de vértebras axis (C2) de población mexicana contemporánea, obtenidos de las colecciones de piezas osteológicas de los Departamentos de Anatomía Humana de las Facultades de Medicina y Odontología de la U.A.N.L. y del Laboratorio de Antropología Física del Departamento de Anatomía de la Facultad de Medicina de la U.N.A.M. Se excluyeron aquellas piezas en las que se observó daño o anormalidades evidentes en su estructura. Las mediciones de las vértebras se efectuaron bilateralmente utilizando un vernier digital milimétrico con una precisión de
0,01 mm (Mitutoyo Digimatic w/Absolute Encoders- Series 500). Todas las mediciones se reportaron en $\mathrm{mm}$.

Los parámetros morfométricos evaluados en el presente estudio son los de mayor importancia clínica para la estabilización atlantoaxial mediante los distintos abordajes quirúrgicos existentes y fueron los siguientes (Fig. 1):

A. Distancia entre el margen lateral y medial del istmo de la pars interarticular, a nivel de su punto más estrecho.

B. Distancia entre los márgenes superior e inferior del istmo de la pars interarticular, a nivel de su punto más estrecho.

C. Distancia vertical entre el punto de unión del proceso odontoideo con el cuerpo vertebral de C2 por su cara anterior y el margen libre superior del proceso odontoideo.

D. Distancia horizontal entre ambos márgenes laterales del proceso odontoideo de $\mathrm{C} 2$ a nivel de la mitad de la faceta articular de la misma por su cara anterior.

E. Distancia entre ambos márgenes laterales de la faceta articular del proceso odontoideo de $\mathrm{C} 2$, a nivel de su punto medio.

F. Distancia entre los márgenes anterior y posterior del cuerpo vertebral de $\mathrm{C} 2$ a nivel de la línea mediana, por su cara inferior.

G. Distancia entre ambos márgenes laterales del cuerpo vertebral de $\mathrm{C} 2$, por su cara inferior.

H. Distancia entre el margen inferior del cuerpo vertebral de C2 en su cara anterior y el punto de unión del margen superior del cuerpo vertebral de C2 con el proceso odontoideo.

I. Distancia entre el margen superior e inferior de la lámina de C2 a nivel de su punto medio.

J. Distancia entre los márgenes anterior y posterior de la lámina de $\mathrm{C} 2$.

Análisis Estadístico. Fue realizado utilizando el programa computacional Microsoft Excel 2013 para Windows XP. Se determinó la media, desviación estándar, resultado mínimo y resultado máximo para cada parámetro morfométrico estudiado, todos los resultados son expresados en $\mathrm{mm}$.

Previo a la realización de las morfometrías se realizó un análisis interobservador para la estandarización de la técnica y los puntos de medición utilizando una prueba de $\mathrm{t}$ student de dos colas para determinar la significancia de las diferencias entre las mediciones de los dos observadores, considerando como significativo un valor de p inferior a 0,05 .

Consideraciones Éticas. El presente protocolo fue aprobado por el Comité de Ética y el Comité de Investigación de la Facultad de Medicina de la U.A.N.L. No existen ganancias financieras o comerciales por la realización del presente estudio, por lo que los autores declaran no tener ningún tipo de conflicto de interés. 

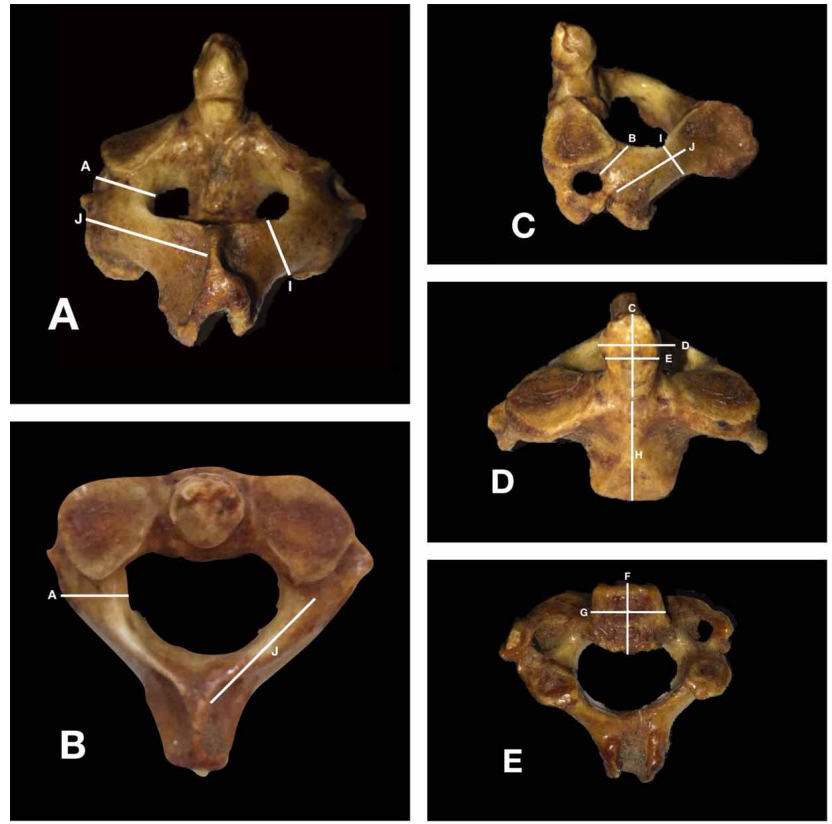

\section{RESULTADOS}

El presente estudio analizó de manera cuantitativa las características morfométricas de mayor importancia quirúrgica de la vértebra axis (C2), estas se muestran en la Tabla I. Los resultados de las pruebas de t student arrojaron resultados no significativos $(p>0,05)$ para las diferencias
Fig. 1. Imagen representativa de las morfometrías del axis realizadas en el presente estudio. A. Distancia entre el margen lateral y medial del istmo de la pars interarticular, a nivel de su punto más estrecho. B. Distancia entre el margen superior e inferior del istmo de la pars interarticular, a nivel de su punto más estrecho. C. Distancia vertical entre el punto de unión del proceso odontoides con el cuerpo vertebral de $\mathrm{C} 2$ por su cara anterior y el margen libre superior del proceso odontoides. D. Distancia horizontal entre ambos márgenes laterales del proceso odontoides de $\mathrm{C} 2$ a nivel de la mitad de la faceta articular de la misma por su cara anterior. E. Distancia entre ambos márgenes laterales de la faceta articular del proceso odontoides de C2, a nivel de su punto medio. F. Distancia entre el margen anterior y posterior del cuerpo vertebral de $\mathrm{C} 2 \mathrm{a}$ nivel de la línea media, por su cara inferior. G. Distancia entre ambos márgenes laterales del cuerpo vertebral de $\mathrm{C} 2$, por su cara inferior. H. Distancia entre el margen inferior del cuerpo vertebral de $\mathrm{C} 2$ en su cara anterior y el punto de unión del margen superior del cuerpo vertebral de $\mathrm{C} 2$ con el proceso odontoides. I. Distancia entre el margen superior e inferior de la lámina de $\mathrm{C} 2$ a nivel de su punto medio. J. Distancia entre el margen anterior y posterior de la lámina de $\mathrm{C} 2$.

interobservador en todos los parámetros morfométricos estudiados.

En la Tabla I se muestran los parametros mofrométricos evaluados en el presente estudio.

Tabla I. Resultados de los parámetros morfométricos evaluados en el presente estudio (Todos los resultados expresados en mm). A. Distancia entre el margen lateral y medial del istmo de la pars interarticular, a nivel de su punto más estrecho. B. Distancia entre el margen superior e inferior del istmo de la pars interarticular, a nivel de su punto más estrecho. C. Distancia vertical entre el punto de unión del proceso odontoides con el cuerpo vertebral de C2 por su cara anterior y el margen libre superior del proceso odontoides. D. Distancia horizontal entre ambos márgenes laterales del proceso odontoides de C2 a nivel de la mitad de la faceta articular de la misma por su cara anterior. E. Distancia entre ambos márgenes laterales de la faceta articular del proceso odontoides de C2, a nivel de su punto medio. F. Distancia entre el margen anterior y posterior del cuerpo vertebral de C2 a nivel de la línea media, por su cara inferior. G. Distancia entre ambos márgenes laterales del cuerpo vertebral de $\mathrm{C} 2$, por su cara inferior. H. Distancia entre el margen inferior del cuerpo vertebral de C2 en su cara anterior y el punto de unión del margen superior del cuerpo vertebral de C2 con el proceso odontoides. I. Distancia entre el margen superior e inferior de la lámina de C2 a nivel de su punto medio. J. Distancia entre el margen anterior y posterior de la lámina de $\mathrm{C} 2$.

\begin{tabular}{ccccc}
\hline $\begin{array}{c}\text { Parámetro } \\
\text { Morfométrico }\end{array}$ & Media & $\begin{array}{c}\text { Desviación } \\
\text { Estándar }\end{array}$ & $\begin{array}{c}\text { Resultado } \\
\text { Mínimo }\end{array}$ & $\begin{array}{c}\text { Resultado } \\
\text { Máximo }\end{array}$ \\
\hline A & 8,69 & $\pm 2,11$ & 6,14 & 17,81 \\
B & 10,82 & $\pm 1,89$ & 6,21 & 16,55 \\
C & 16,90 & $\pm 2,99$ & 13,48 & 20,66 \\
D & 9,99 & $\pm 0,80$ & 7,4 & 11,63 \\
E & 8,44 & $\pm 1,04$ & 4,97 & 10,84 \\
F & 15,11 & $\pm 1,88$ & 10,5 & 20,7 \\
G & 17,93 & $\pm 2,22$ & 14,04 & 25,45 \\
H & 18,54 & $\pm 2,38$ & 8,25 & 23,63 \\
I & 11,53 & $\pm 1,39$ & 3,48 & 14,69 \\
J & 6,10 & $\pm 1,44$ & 11,94 \\
\hline
\end{tabular}




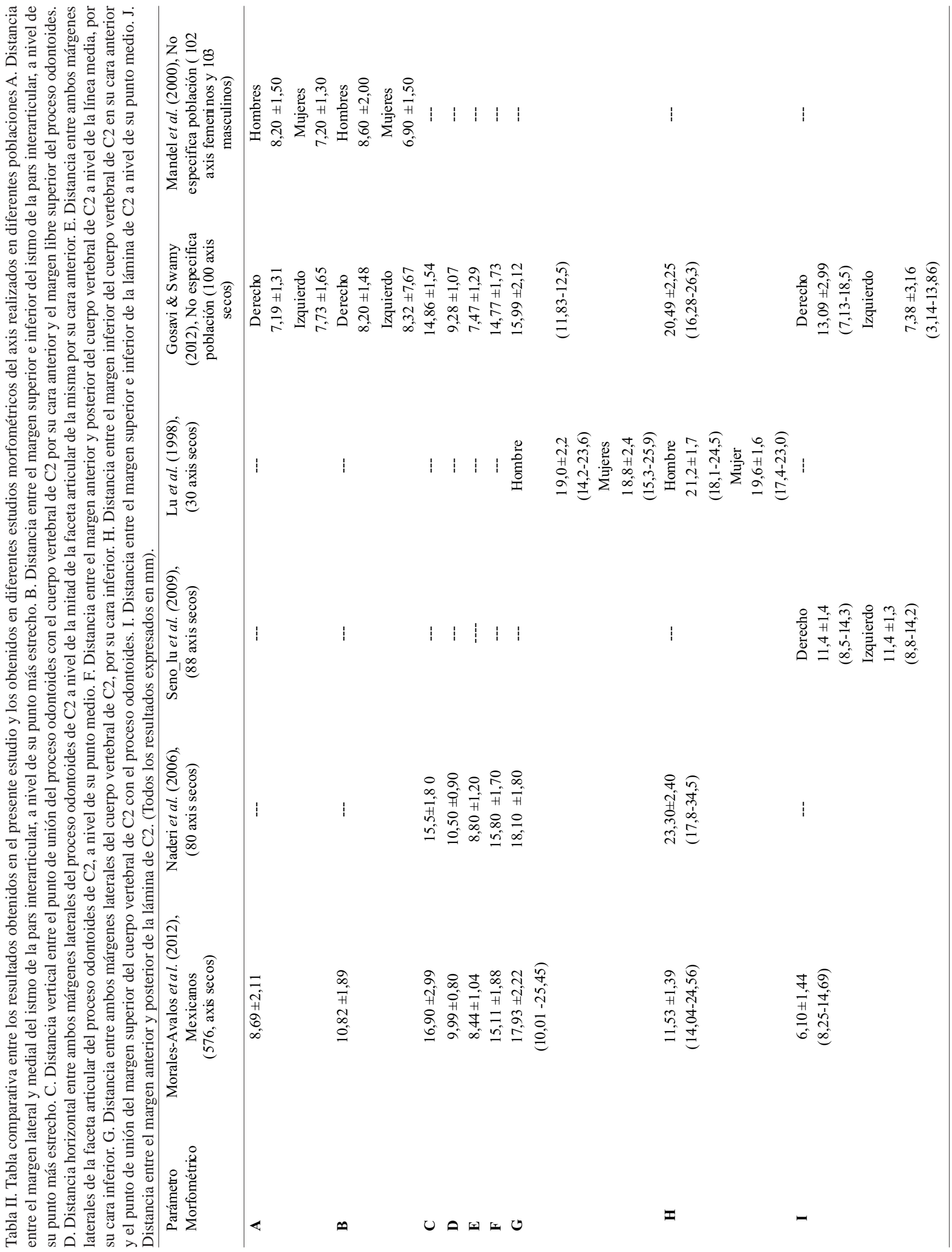




\section{DISCUSIÓN}

La segunda vértebra cervical difiere del resto de las vértebras cervicales porque posee características anatómicas peculiares, las cuales hacen que su abordaje quirúrgico represente un reto para el cirujano. Existen estudios que evalúan las características morfométricas de esta vértebra en diferentes poblaciones, pero no existen, hasta el momento, estudios realizados en población mexicana.

Actualmente existe confusión en la nomenclatura descriptiva de la vértebra axis (C2) debido a su morfología única. El verdadero pedículo anatómico de $\mathrm{C} 2$ se encuentra anterior del proceso articular, uniendo los elementos dorsales del axis con su cuerpo vertebral (Mandel et al., 2000) (Fig. 1).

El estrecho puente de hueso que se encuentra inmediatamente posterior al proceso articular y medial al foramen transverso, es frecuentemente descrito como el pedículo de $\mathrm{C} 2$, sin embargo este representa el istmo de la pars interarticular (Mandel et al.).

Magerl y Seeman indicaron y establecieron la fijación transarticular de C1 y C2 en 1979. Algunas indicaciones para esta técnica, son las lesiones traumáticas de $\mathrm{C} 1$, fractura del proceso odontoideo y/o defectos congénitos de la columna cervical alta (Tan et al., 2003).

Se debe de realizar una adecuada planificación quirúrgica y verificar bien el tamaño de los pedículos, esto con intención de disminuir al mínimo el riesgo de lesión de la arteria vertebral (4-8\% de los casos).

Los resultados obtenidos respecto a la diámetro del istmo de la pars interarticular resultan muy similares a los obtenidos en el estudio de población americana de Mandel et al. Sin embargo los resultados del mismo estudio, respecto a la altura de la pars interarticular resultan inferiores a los obtenidos en el presente estudio. Panjabi et al., en su estudio cadavérico de la vértebra $\mathrm{C} 2$, determinaron que el diámetro del istmo pedicular de C2 varía entre 7 y $9 \mathrm{~mm}$ y la altura del mismo entre 9 y $11 \mathrm{~mm}$. Estas diferencias podrían explicarse a las diferencias poblacionales y a la gran antigüedad de las piezas osteológicas utilizadas en esos estudios.

Mandel et al. determinaron que la colocación de tornillos en la pars interarticular del axis resulta de gran complejidad si el diámetro mínimo de la misma es menor de $5 \mathrm{~mm}$, esto dificultaría la colocación de tornillos de $3,5 \mathrm{~mm}$ bajo visión fluoroscópica. Los resultados de nuestro estudio indican que las características morfométricas del axis en nuestra población parecen adecuadas para este tipo de fijación.
En la Tabla II se concentran los resultados de estudios morfométricos previos del axis.

\section{CONCLUSIONES}

Los parámetros morfométricos de la vértebra axis (C2) en la población mexicana difieren de los reportados en la literatura. El presente estudio evalúa de manera integral los parámetros morfométricos involucrados en la realización de procedimientos quirúrgicos de la vértebra axis (C2).

\section{AGRADECIMIENTOS}

Al Lic. Jaime A. Cisneros Ríos por la ayuda prestada para la preparación del material gráfico presentado en este estudio y al A.F Guillermo Torres por la ayuda técnica proporcionada para la realización del presente estudio.

MORALES-AVALOS, R.; VILLARREAL-GARCIA, F. I.; REQUENA-ARAUJO, P. M.; VÍLCHEZ-CAVAZOS, F.; REYES-FERNÁNDEZ, P. M.; MARTÍNEZ-GUTIERREZ, O.A.; PEÑA-MARTíNEZ, V. M.; GUZMÁN-LÓPEZ, S. \& ELIZONDO-OMAÑA, R. E. Morphometric analysis of the axis for atlantoaxial fixation. Int. J. Morphol., 36(4):1331-1336, 2018.

SUMMARY: The aim of the study was to evaluate the morphometric parameters of the axis vértebra (C2) in the Mexican population involved in the performance of surgical procedures in order to provide essential quantitative data in their surgical approach. A total of 576 axis vertebrae (C2) of contemporary Mexican population were used for this study. The measurements of the vertebrae were made bilaterally using a millimeter digital vernier with an accuracy of 0.01 millimeters (Mitutoyo Digimatic w / Absolute Encoders - Series 500). A total of 576 axis vertebrae (C2) were measured bilaterally, all our measurements were reported in millimeters. The average width of the pedicle was $8.96 \mathrm{~mm}$ with a SD $\pm 2.11 \mathrm{~mm}$. The average height of the pedicle was $10.82 \mathrm{~mm}$ with a SD of $\pm 1.89 \mathrm{~mm}$. The average height of the odontoid process was $16.90 \mathrm{~mm}$ with a SD of $\pm 2.99 \mathrm{~mm}$. The average width of the odontoid process was $9.99 \mathrm{~mm}$ with a SD of $\pm 0.80 \mathrm{~mm}$. The average diameter of the articular facet was $8.44 \mathrm{~mm}$ with a SD of \pm $1.04 \mathrm{~mm}$. The average diameter of the AP of the vertebral body was $15.11 \mathrm{~mm}$ with a SD of $\pm 1.88 \mathrm{~mm}$. The average transverse diameter of the vertebral body was $17.93 \mathrm{~mm}$ with a SD of \pm 2.22 $\mathrm{mm}$. The average height of the vertebral body was $18.54 \mathrm{~mm}$ with a SD of $2.38 \mathrm{~mm}$. The average height of the lamina was $11.53 \mathrm{~mm}$ with a SD of $\pm 1.39 \mathrm{~mm}$. The average width of the lamina was 6.10 $\mathrm{mm}$ with a SD of $\pm 1.44 \mathrm{~mm}$. The results obtained in our 
measurements show a variation with the results of other authors in different studies of osteological pieces and studies of the axis image (C2), which suggests the use of techniques and special measures for the atlantoaxial stabilization of the Mexican population in order to reduce the risk of damage to neurovascular structures.

KEY WORDS: Anatomy; Morphometry; Axis; C2; Atlantoaxial junction; Mexico.

\section{REFERENCIAS BIBLIOGRÁFICAS}

Dickman, C. A.; Crawford, N. R.; Brantley, A. G. \& Sonntag, V. K. Biomechanical effects of transoral odontoidectomy. Neurosurgery, 36(6):1146-52, 1995.

Dickman, C. A.; Sonntag, V. K.; Papadopoulos, S. M. \& Hadley, M. N. The interspinous method of posterior atlantoaxial arthrodesis. J. Neurosurg., 74(2):190-8, 1991.

Fried, L. C. Atlanto-axial fracture-dislocations. Failure of posterior C. 1 to C.2 fusion. J. Bone Joint Surg. Br., 55(3):490-6, 1973

Gosavi, S. \& Swamy, V. Morphometric study of the Axis vertebra. Eur. J. Anat., 16(2):98-103, 2012.

Hanson, P. B.; Montesano, P. X.; Sharkey, N. A. \& Rauschning, W. Anatomic and biomechanical assessment of transarticular screw fixation for atlantoaxial instability. Spine (Phila Pa 1976), 16(10):1141-5, 1991.

Heller, J. G.; Amrani, J. \& Hutton, W. C. Transverse ligament failure: a biomechanical study. J. Spinal Disord., 6(2):162-5, 1993.

Jeanneret, B. \& Magerl, F. Primary posterior fusion C1/2 in odontoid fractures: indications, technique, and results of transarticular screw fixation. J. Spinal Disord., 5(4):464-75, 1992.

Lu, J.; Ebraheim, N. A.; Yang, H.; Heck, B. E. \& Yeasting, R. A. Anatomic considerations of anterior transarticular screw fixation for atlantoaxial instability. Spine (Phila Pa 1976), 23(11):1229-35, 1998.

Mandel, I. M.; Kambach, B. J.; Petersilge, C. A.; Johnstone, B. \& Yoo, J. U. Morphologic considerations of $\mathrm{C} 2$ isthmus dimensions for the placement of transarticular screws. Spine (Phila Pa 1976), 25(12):15427, 2000.

Morales-Avalos, R.; Re Elizondo-Omaña, R. E.; Vílchez-Cavazos, F.; Martínez-Ponce de León, A. R.; Elizondo-Riojas, G.; Delgado-Brito, M.; Cortés-González, P.; Guzmán-Avilán, R. I.; Pinales-Razo, R.; de la Garza-Castro, O. \& Guzmán-López, S. Vertebral fixation with a transpedicular approach. Relevance of anatomical and imaging studies. Acta. Ortop. Mex., 26(6):402-11, 2012.

Naderi, S.; Arman, C.; Güvençer, M.; Korman, E.; Senoglu, M.; Tetik, S. \& Arda, M. N. Morphometric analysis of the $\mathrm{C} 2$ body and the odontoid process. Turk. Neurosurg., 16(1):14-8, 2006.

Panjabi, M.; Dvorak, J.; Crisco, J. J. 3rd; Oda, T.; Wang, P. \& Grob, D. Effects of alar ligament transection on upper cervical spine rotation. $J$. Orthop. Res., 9(4):584-93,1991.

Penning, L. \& Wilmink, J. T. Rotation of the cervical spine. A CT study in normal subjects. Spine (Phila Pa 1976), 12(8):732-8, 1987.

Senoglu, M.; Ozbag, D. \& Gümüsalan, Y. C2 intralaminar screw placement: a quantitative anatomical and morphometric evaluation. Turk. Neurosurg., 19(3):245-8, 2009.

Tan, M.; Wang, H.; Wang, Y.; Zhang, G.; Yi, P.; Li, Z.; Wei, H. \& Yang, F. Morphometric evaluation of screw fixation in atlas via posterior arch and lateral mass. Spine (Phila Pa 1976), 28(9):888-95, 2003.
Dirección para correspondencia:

Dr. Rodolfo Morales Avalos

Servicio de Ortopedia y Traumatologia

Hospital Universitario "Dr. Jose Eleuterio González"

Universidad Autónoma de Nuevo León (U.A.N.L.)

Ave. Madero s/n Col. Mitras Centro

Monterrey, Nuevo León

C.P. 64460

MÉXICO

Email: rodolfot59@hotmail.com

Recibido : 12-04-2018

Aceptado: 26-06-2018 